\title{
The effects of processing speed and menry span on working menory
}

\begin{tabular}{|l|l|}
\hline 著者 & Kuni m M t sunobu, Koj i ma Har uyuki \\
\hline 著者別表示 & 國見 充展 \\
\hline $\begin{array}{l}\text { j our nal or } \\
\text { publ i cat i on t i t l e }\end{array}$ & $\begin{array}{l}\text { Ger oPsych: The Jour nal of Ger ont opsychol ogy } \\
\text { and Ger i at r i c Psychi at ry }\end{array}$ \\
\hline vol une & 27 \\
\hline nunber & 3 \\
\hline page range & $109-114$ \\
\hline year & 2014 10-01 \\
\hline URL & ht t p: //doi . or g/10. 24517/00000166 \\
\hline
\end{tabular}


Short Report

The effects of processing speed and memory span on working memory

Mitsunobu Kunimi (National Center for Geriatrics and Gerontology)

Haruyuki Kojima (Kanazawa University)

Contact; Mitsunobu Kunimi (Ph.D)

National Center for Geriatrics and Gerontology

35 Gengo Morioka-cho Obu city Aichi

$474-8522$

TEL $+81562445651(5633)$

FAX +81562467827

kunimi@ncgg.go.jp 
Abstract

This study examined the processing speed and memory span of young adults and older people using tasks based on the

Wechsler Adult Intelligence Scale-III (WAIS-III, Wechsler, 1997). By comparing the data obtained from these tasks, we

examined the effects of processing speed and memory span on working memory (WM). In addition, this study examined how presentation modality and the subject's age are related to WM. Multiple regression analysis of the effect of memory span for each presentation modality used processing time as a factor to predict the WM span of various age groups. The result was two equations for predicting WM span. According to these equations, WM is negatively correlated with "age group" and "processing time," and positively correlated with "memory span." Memory span and processing speed were found to have similar impacts on WM, regardless of the presentation modality. However, our results suggested that visual WM and auditory WM are different functions, and that auditory WM is more strongly affected by memory span than visual WM.

Keywords

Aging, Cognition, Working Memory 
Working memory is a function that involves the temporary storage and processing of information as a means of supporting complex cognitive processes. According to the working memory model developed by Baddeley (1986), the central executive function is responsible for the distribution of attentional resources and monitoring, and the visuospatial sketchpad and phonological loop act as slave systems. The phonological loop handles verbal information and acts as an inner ear or inner voice during the rehearsal of temporarily stored information. On the other hand, the visuospatial sketchpad corresponds to the inner eye and can retain non-verbal visual information that cannot be retained by phonological rehearsal. These slave systems are assumed to function co-operatively in the temporary storage of information. In addition to the retention of information, working memory is also involved in the simultaneous processing of information.

Age can affect working memory. Studies of age-related changes in working memory have gained attention because such changes can have strong effects on commonly used cognitive functions. For example, when the performances of a group of 60-70-year-old subjects and a group of young adults in a task that involved sorting strings of presented letters into alphabetical order were compared, it was found that the groups exhibited different performance levels during both the forward and reverse cascade sorting tasks (Craik, 1986). In addition, West (1999) investigated the effects of aging on selective attention and working memory processing and found that both the young and old groups made more errors when distractors were introduced; however, in the older adults the distractors also had a significant influence on encoding and retention. Furthermore, Meguro et al (2000) demonstrated age-related differences in performance during a reading span 
task, and Dobbs \& Rule (1987) reported an age-related decline in working memory performance. All of these studies demonstrated marked age-related differences in working memory. Accordingly, the effect of age must be considered in studies of working memory.

Working memory is considered to be affected by memory span (Hasher \& Zacks, 1988) and processing speed (Salthouse, 1991, 1996). Although several studies were published targeting the influence of memory span and processing speed on working memory performance in aging (e.g., Fisk \& Warr, 1996; Foos, 1989; Salthouse \& Meinz, 1995), the direct comparison of the effects of age on memory span and the processing speed of working memory is highly controversial. So, in this study we attempted to predict working memory from the results of standardized tests and the subject's age.

In this study the digit span task and the digit-symbol substitution task used as the task which is included in the standardized Wechsler Adult Intelligence Scale-III (WAIS-III, Wechsler, 1997). In the digit span task, the subjects are instructed to remember an orally presented string of digits and then recite it anterogradely (forward cascade) or retrogradely (backward cascade). Baddeley $(1986,2000)$ subsequently modified the working memory model based on the effects of phonological loop order on performance in cascade tasks that required the short-term retention of digits, which can be used as a measure of the capacity of the central executive to invert the cascade of processes during retrograde recital. It is considered that forward cascade tasks can be used to estimate simple memory span, and backward cascade tasks can be used to measure working memory span. In addition, the digit-symbol substitution task was used as a measure 
of processing speed. In this task, the digits 1-9 are each assigned a symbol. The subjects are then presented with a long list of digits and have to write as many of the corresponding symbols as they can within a set time period. The number of correct symbols produced within the allowed time is then evaluated. This task requires speed, accuracy, handwriting skills, and agility; i.e., it involves a mix of physical and cognitive functions. The subjects are also required to perform an auxiliary task in order to eliminate inter-individual differences in their ability to physically encode the symbols. In the auxiliary task, the subjects are only required to transcribe the symbols; i.e., the symbols are not paired with particular numbers. By comparing the data obtained in these tasks, it is possible to examine the effects of processing speed and memory span on working memory.

In addition, the modality used to present stimuli might also affect working memory performance. One example of this is the modality effect. When memorizing language stimuli, a stimulus modality-dependent difference in recall performance is observed, and this is known as the modality effect. For example, when individuals were asked to immediately recall a list of words or letters that had just been presented to them, better performance was observed when the stimuli were presented in an auditory rather than visual manner. Such effects have been reported in many studies (e.g., Gibson \& Bahrey, 2005; Pilotti, Bergman, Gallo, Sommers, \& Roediger, 2000; Hayman \& Rickard, 1995; Schacter \& Graf, 1989). This suggests that different information processing pathways are involved in visual and auditory memory (Hamada, 1986). Although Reuter-Lorenz et al (2000) have revealed asymmetries in the lateral organization of verbal and spatial working memory, the effects of aging on the modality of working memory are largely unknown; e.g., how stimulus 
presentation modality affects the age-related decline of working memory span. Cognitive functions among the elderly are known to exhibit large inter-individual differences so the overall effects of aging on working memory span are difficult to demonstrate. However, by modeling the effects of cognitive function among individuals using tasks that assess memory span and processing speed it is possible to predict the effect of aging on working memory for each presentation modality.

The study has the two goals: First, if and how different cognitive processes influence working memory performance in young and older adults. Second, if results differ depending on the presentation type of the task material. Age-related reductions in working memory have been explained by decreases in memory span with age (Hasher \& Zacks, 1988). Memory span represents the ability of an individual to temporarily retain information that requires at least a certain amount of holding capacity. Another explanation presented by Salthouse $(1991,1996)$ was that age-related reductions in processing speed could cause a decline in overall task performance. It was suggested that reductions in processing speed caused by input/output delays, changes in strategy execution, an increased cognitive burden, and/or slower information processing result in noise and/or increases in neurotransmission (Salthouse, 1985). Therefore, age-related changes in memory span and processing speed might affect working memory independently or in a complex manner.

\section{Methods}

Participants. The participants were 72 young adults $(18-21$-years-old, mean age $=20.5, \mathrm{SD}=0.9)$ and 74 elderly adults $(65-84$-years-old, mean age $=73.0, \mathrm{SD}=5.1)$. The young group consisted of 36 males and 36 females, and the elderly group consisted of 34 males and 40 females. All of the participants in the young group were college students. 
Elderly people in Kanazawa city and the suburban area voluntarily participated in this study. They were recruited through several local community associations in the city. Prior to the study, they had a medical checkup by a physician, and participants who diagnosed healthy enough participated in the study. They also did not have any feeling of illness or any problem in daily life during the time of participation. They all had normal or corrected-to-normal vision. Some of them, who had hearing difficulty, used hearing aids. The elderly group was screened with the Mini-Mental State Examination (MMSE) and achieved a mean score of $28.1(\mathrm{SD}=1.3)$. The entire experiment was explained carefully to each subject prior to their written informed consent being obtained.

Apparatus. A Windows-based personal computer (Toshiba, Dynabook Satellite J40) was used in conjunction with a 15-inch color LCD monitor (TFT color LCD monitor) and psychological experiment control software (Cedrus, SuperLabPro) to present the stimuli in Digit span test. A4 paper was used for the digit symbol substitution task. Numbers from 1-9 were assigned meaningless symbols (e.g., 1:/ -, 2: / $\perp_{\ldots}$ 7:/ $\left.\Lambda, 8: / \mathrm{X}, 9: /=\right)$ at the top of the page, and a random string of digits was written at the bottom of the page.

Procedure. All of the tasks were based on the WAIS-III.

Digit span test. The digit span test is used to measure the number storage capacity of an individual's working memory.

In this study, it was expanded by adding a visual stimulus presentation condition. The experimental factors included presentation order (forward or backward cascade), age group (young or elderly group), and presentation modality (auditory or visual). The participants were presented with a series of digits and were asked to recall them immediately 
after the series had been presented. The participants had to recall the digits orally in a forward (forward condition) or backward cascade (backward condition). If they did this successfully, then they were given a longer list to recite. In the visual presentation experiment, the digits were presented visually on a computer screen one by one. In the auditory presentation experiment, the experimenter read the numbers aloud in order. In both conditions, the digits were presented at the speed of one per second. The number of single-digit increments performed before the participant made an error was recorded (Maximum length: 10). Each list length was only shown once. The order of the task application was randomized. Age group (young vs. elderly) was considered to be a between participant factor, and the task (forward vs. backward condition) and modality (auditory vs. visually presentation) were treated as a within participant factor.

Processing speed test. The tasks used to measure processing speed were based on the WAIS digit-symbol substitution task. In this task, the digits 1-9 were each assigned a symbol. The subjects were then presented with a long list of digits and had to write as many of the corresponding symbols as they could within the set time period ( 30 seconds). The number of correct symbols produced within the allowed time was then evaluated. A practice trial involving seven digits was administered first. After completing the digit symbol task, the participants were given the auxiliary task. The procedure for the auxiliary task was the same as that for the digit-symbol task, but no numbers were provided at the bottom of the page; instead, the participants was just instructed to copy the codes from the top of the page.

Data analysis. Dependent behavioral data variables were the number of recall (in Digit span test) and the coding speed (in Processing speed test). Statistical significance was evaluated by three-way analysis of the variants (ANOVA; task $\times$ 
age group $\times$ presentation modality) in Digit span test. When main effects were detected, a Bonferroni post-hoc multiple comparison analysis was performed. Subsequently, we attempted to predict working memory from memory span and processing speed after adding age as a variable. Age was regarded as dummy variable. The relationships between age group, memory span, working memory span, and processing speed were evaluated with multiple regression analysis, and equations for predicting working memory were calculated for each modality.

\section{Results}

Figure 1 shows the results of the digit span task for the young and elderly groups. Overall, the young group performed better than the elderly group in forward and backward tasks. Both age groups achieved higher scores in the forward cascade task than in the reverse cascade task. In a three-factor mixed analysis of variance (task $\times$ age group $\times$ presentation modality), task $(F(1,144)=363.748, p<0.001)$ and age group $(F(1,144)=128.319, p<0.001)$, but not presentation modality $(F(1,144)=3.263$, ns), were extracted as main effects. In addition, significant interactions were found for presentation modality $\times$ task $(F(1,144)=6.991, p<0.001)$ and age group $\times$ task $\times$ modality presentation $(F(1,144)=$ $5.584, p<0.05)$. The age group $\times$ presentation modality $(F(1,144)=0.006, n s)$ and age group $\times$ task $(F(1,144)=2.270$, $n s)$ interactions were not significant. Presentation modality was found to have a simple main effect on the influence of task on performance in the digit span task $(p<0.01)$. As the presentation modality $\times \operatorname{task} \times$ age group interaction had been demonstrated to be significant, a simple test of each main effect was conducted. Simple main effects of age group 
and task were observed. Presentation modality was found to have a simple main effect on the influence of task on

performance in the forward cascade task in young group only. (all $p<0.001)$.

For the digit-symbol task, the results were calculated as coding speed; i.e., as the amount of time required to transcribe each symbol. The digit-symbol task involved both transcription and coding, while the auxiliary task only involved transcription. The difference between a subject's coding speeds in the digit-symbol and auxiliary tasks is considered to reflect processing speed. The young group exhibited a significantly faster processing speed $($ mean $=0.58 ; S D=0.13)$ than the elderly group $($ mean $=1.28 ; S D=0.70)(F(1,144)=69.689, p<0.001)$.

Next, we attempted to predict working memory from memory span and processing speed after adding age as a variable. Age is used as dummy variable. The relationships between age group, memory span, working memory span, and processing speed were evaluated with multiple regression analysis, and equations for predicting working memory were calculated for each modality. In the equation for the auditory modality, which is shown below, "A" represents age group. In addition, hearing memory span (aM) was obtained from the forward cascade task results, and auditory working memory span $(\mathrm{aW})$ was derived from the reverse cascade task results. The processing time $(\mathrm{S})$ was calculated from the results of the digit-symbol task. As a result, the following regression equation was obtained:

$$
\mathrm{aW}=-0.37 \mathrm{~A}+0.35 \mathrm{aM}-0.33 \mathrm{~S}+2.68
$$

The equation demonstrated a significant fit with the relationship between age, working memory, and processing speed in the auditory modality conditions $(F(3,145)=34.320, p<0.001)$. In the multiple regression analysis, these factors 
exhibited a correlation coefficient of $R=0.658$ (coefficient of determination, $R^{2}=0.433$ ) in the auditory presentation

conditions. The results of the analysis are presented in Table 1. Memory span was found to be a significant predictor of

$\mathrm{aW}$ at the $5 \%$ level, while processing time was found to be a significant predictor at the $1 \%$ level.

In the equation for the visual modality, visual memory span (vM) was calculated from the results of the forward

cascade task, and visual working memory span $(\mathrm{vW})$ was derived from those of the reverse cascade task. The equation

was as follows:

$$
\mathrm{vW}=-1.02 \mathrm{~A}+0.25 \mathrm{vM}-0.25 \mathrm{~S}+3.61
$$

The equation demonstrated a significant fit with the relationship between age, working memory, and processing speed in the visual modality conditions $\left(R=0.631\right.$, coefficient of determination: $\left.R^{2}=0.398, F(3,145)=35.505, p<0.001\right)$. The results of the regression analysis are shown in Table 2. "Age group" and "memory span" were both found to be significant predictors of vW at the $1 \%$ level. For both the auditory and visual presentation modalities, "age group" and "processing time" exhibited negative correlations with vW, while "memory span" was positively correlated with it.

\section{Discussion}

In this study, we compared the processing speed, memory span, and working memory span of young and elderly

people using standardized tasks. The digit-symbol task showed that aging affects processing speed. In addition, the cascade tasks indicated that aging also affects short-term information retention. Presentation modality has been reported 
to affect working memory in many previous studies (e.g., Gibson \& Bahrey, 2005; Pilotti et al., 2000; Hayman \& Rickard, 1995; Schacter \& Graf, 1989), and it was found to interact with other factors in the current study. Research on dual task paradigms as well as task switching paradigms has been dealt with the interference, and therefore drops in performance arising because of input-output compatibilities (Stephan \& Koch, 2010; Haseltine \& Wifall, 2011). In this study, the significant difference between the presentation modality is observed in the condition of the forward cascade task in young group only. In the backward cascade task, the end of the digit list can be drawn from iconic memory. Therefore, the interference of modality disappeared apparently. However, in the elderly group an inter-modality performance difference was not observed in the condition of the forward cascade task, which might have caused by a perceived time delay (Lindenberger \& Baltes, 1994) due to the subjects’ reduced sensory memory capacity (Walsh \& Thompson, 1978).

Further multiple regression analysis of the effect of each presentation modality, in which processing time was included as a factor, was performed in an attempt to derive equations that could be used to predict working memory span in each age group. As a result, two equations for predicting working memory span were developed. When the standardized coefficients ( $\beta$ ) used in these equations were examined, it was revealed that both "age group" and "processing time" demonstrated negative correlations with working memory, while "memory span" was positively correlated with it. In other words, when memory span or processing speed increased, so did working memory span. Thus, memory span and processing speed were found to have approximately the same impact on working memory, regardless of the presentation modality. However, in the auditory presentation conditions the standardized coefficient for memory span was larger, and 
those for age and processing speed were equivalent. On the other hand, in the visual presentation conditions age exhibited the largest standardized coefficient, followed by memory span and processing speed. As the constant in this equation was also large, other factors such as perception and attention might strongly affect working memory in the visual presentation conditions. In the auditory presentation conditions, "processing speed" was found to significantly affect auditory working memory. As noted earlier, auditory working memory is dependent on the echoic retention time, which can affect both task performance and processing speed. Visual working memory and auditory working memory might be qualitatively different and exist independently in the brain (Hamada, 1986), which could be due to differences in the input paths for auditory and visual memory.

This study aimed to directly examine the effects of aging on memory span and the processing speed of working memory using standardized tests. Direct comparisons of the sizes of the effects of the explanatory variables (processing speed and memory span) on the explained variable (working memory) are crucially important. This study suggested that visual working memory and auditory working memory are different functions and that auditory working memory is affected more strongly by memory span than visual working memory. Considering the inter-individual differences in the cognitive functions of the elderly, it is difficult to draw definitive conclusions regarding the effects of aging on working memory span. In addition, it should be noted that other results might be found when using different tasks. For example, Bopp and Verhaghen (2005) showed in their meta-analysis that age effects for such "reordering tasks" fall between simple storage span tasks (e.g., digit span forward) and working memory span tasks (e.g., reading span). Furthermore, the aging 
effect remains in the visual conditions, though the age difference in working memory is not significant when processing speed and memory span are entered as predictors in the analysis in the auditory condition. This might be the evidence for different modality has different aging effect. Other analysis method might reveal how the different variables influence the age effect (i.e., hierarchical regression analysis). However, based on measurements of cognitive processing speed and memory span for different presentation modalities obtained using standardized tests we have demonstrated that it is possible to predict working memory whilst taking the effects of age into account.

Finally, this study cannot control participant's educational history. Previous study which was investigated the effects of demographic variables on the performances of the Benton Visual Retention Test (BVRT; Sivan, 1992)) suggested that both nonverbal memory and constructional ability are influenced by age and education (Seo, Lee, Choo, Youn, Kim, Jhoo, Suh, Paek, Jun, \& Woo, 2007). The selection of participants into the samples is different, and age differences are confounded with cohort differences. For the contention will be make clear, the control of demographic variables is future agenda. 


\section{References}

Baddeley, A. D. (1986). Working memory. New York: Oxford University Press.

Baddeley, A. D. (2000). The episodic buffer: a new component of working memory? Trends in cognitive Sciences, 4 , 417-423.

Bopp, K. L., \& Verhaeghen, P. (2005). Aging and verbal memory span: A meta-analysis. Journal of Gerontology: B Series. 60, 223-233.

Craik, F. I. M. (1986). Two components in free recall. Journal of Verbal Learning and Verbal Behavior, 7, 996-1004.

Dobbs, A. R. \& Rule, B. G. (1989). Adult Age Differences in Working Memory. Psychology and Aging, 4, 500-503.

Fisk, J. E., \& Warr, P. (1996). Age and working memory: The role of perceptual speed, the central executive, and the phonological loop. Psychology and Aging, 11, 316-323.

Foos, P.W. (1989). Adult age differences in working memory. Psychology and Aging, 4, 269-275

Gibson, J. M., \& Bahrey, R. (2005). Modality-specificity effects in priming of visual and auditory word-fragment completion. Journal of General Psychology, 132, 117- 137.

Hazeltine, E., \& Wifall, T. (2011). Searching working memory for the source of dual-task costs. Psychological Research, 75(6), 466-475.

Hayman, C. A. G., \& Rickard, C. (1995). A dissociation in the effects of study modality on tests of implicit and explicit memory. Memory and Cognition, 23, 95-112. 
Hamada, J (1986). Interaction between visual and auditory memory for random digit series under forward and backward rehearsal/recall conditions, Japanese journal of psychonomic science 5, 55-61.

Hasher, L., \& Zacks, R. T. (1988). Working memory, comprehension and aging: A review and a new view. Psychology of Learning and Motivation, 22, 193-225.

Lindenberger, U., \& Baltes, P. B., (1994). Sensory functioning and intelligence in old age: a strong connection. Psychological Aging. 9, 339-55.

Meguro, Y., Fujii, T., Yamadori, A., Tsukiura, T., Suzuki, K., Okuda, J., \& Osaka, M. (2000). The nature of age-related decline on the reading span task. Journal of Clinical and Experimental Neuropsychology, 22, 391-398.

Pilotti, M., Bergman, E. T., Gallo, D, A., Sommers, M., \& Roediger, H. L., III (2000). Direct comparison of auditory implicit memory tests. Psychonomic Bulletin and Review, 7, 357-353.

Reuter-Lorenz, P. A., Jonides, J., Smith, E.E., Hartley, A., Miller, A., Marshuetz, C., \& Koeppe, R.A. (2000). Age differences in the frontal lateralization of verbal and spatial working memory revealed by PET. Jounal of Cognitive Neuroscience. 12, 174-87.

Salthouse, T. A. (1985). Speed of behavior and its implications for cognition. In J. E. Birren \& K. W. Schaie (Eds.), Hand book of the psychology of aging (2nd edition, pp.400-426). New York: VanNostrand Reinhold.

Salthouse, T. A. (1991). Theoretical perspective on cognitive aging. Hillsdale, NJ: Erlbaum. 
Salthouse, T. A. (1996). The processing-speed theory of adult age differences in cognition. Psychological Review, 103, 403-428.

Salthouse, T.A., \& Meinz, E.J. (1995). Aging, inhibition, working memory, and speed. Journal of Gerontology: Psychological Sciences. 50B, 297-306.

Schacter, D. L., \& Graf, P. (1989). Modality specificity of implicit memory for new associations. Journal of Experimental Psychology: Learning, Memory, and Cognition, 15, 3-12

Seo, E. H., Lee, D, Y., Choo, I. H., Youn, J. C., Kim, K. W., Jhoo, J. H., Suh, K. W., Paek, Y. S., Jun, Y. H., \& Woo, J. I. (2007). Performance on the Benton Visual Retention Test in an Educationally Diverse Older Population. Journal of Gerontology, 62, 191-193.

Sivan, A. D. (1992). Benton Visual Retention Test (5th ed.). New York: The Psychological Corporation.

Stephan, D. N., \& Koch, I. (2010). Central cross-talk in task switching: Evidence from manipulating input-output modality compatibility. Journal of Experimental Psychology: Learning, Memory, and Cognition, 36(4), $1075-1081$.

Walsh, D. A., \& Thompson, L. W. (1978). Age differences in visual sensory memory. Journal of Gerontology, 33, 383-387.

Wechsler D. (1997). Wechsler Adult Intelligence Scale-Third Edition. San Antonio: The Psychological Corporation West, R (1999) Visual distraction working memory, and aging Memory \& cognition 6, 1064-1072. 
West, R. L., \& Boatwright, L. K., (1983). Age differences in cued recall and recognition under varying encoding and retrieval conditions. Experimental Aging Research, 9, 185-189. 


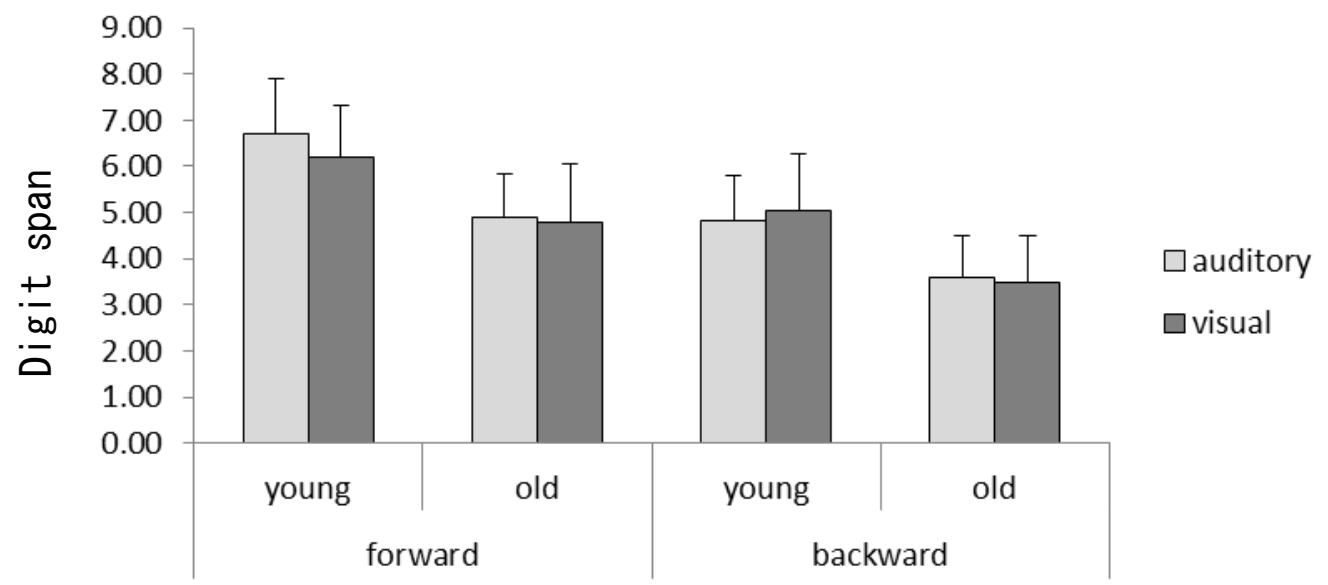

Figure 1. The results of the digit span task in the young and elderly groups. The young group achieved higher scores than the elderly group in both tasks. Both age groups achieved higher scores for the forward cascade task than for the reverse cascade task. 
Table 1. Summary of the results of the multiple regression analysis performed in the auditory conditions

\begin{tabular}{cccc}
\hline variable & standardized coefficent $(\beta)$ & $t$ & $p$ value \\
\hline (constant) & & 5.707 & 0.000 \\
age & -0.165 & -1.810 & 0.072 \\
processing speed & -0.180 & -2.329 & 0.021 \\
memory span & 0.430 & 5.215 & 0.000
\end{tabular}

$\mathrm{R}=0.658$, coefficient of determination $\mathrm{R}^{2}=0.433, \mathrm{~F}(3,145)=34.320, \mathrm{p}<0.001$ 
Table 2. Summary of the results of the multiple regression analysis performed in the visual conditions

\begin{tabular}{|c|c|c|c|}
\hline variable & standardized coefficent $(\beta)$ & $t$ & $p$ value \\
\hline (constant) & & 6.904 & 0.000 \\
\hline age & -0.375 & -4.433 & 0.000 \\
\hline processing speed & -0.114 & -1.391 & 0.166 \\
\hline memory span & 0.260 & 3.321 & 0.001 \\
\hline
\end{tabular}

$\mathrm{R}=0.631$, coefficient of determination $\mathrm{R}^{2}=0.398, \mathrm{~F}(3,145)=35.505, \mathrm{p}<0.001$ 